\title{
Freely Available Software for 3D RF Ultrasound
}

\author{
Graham Treece^, Richard Prager, and Andrew Gee \\ Dept. Engineering, Univ. Cambridge, Cambridge, UK, CB2 1PZ, gmt11@cam.ac.uk
}

\begin{abstract}
We present a 3D radio frequency (RF) ultrasound (US) system capable of acquiring real-time $\mathrm{RF}$ data at a sustained rate of 30-60 frames per second with $60 \mathrm{~dB}$ real dynamic range and typical phase error of only $\pm 2.3^{\circ}$ at $6 \mathrm{MHz}$. The software is freely available to other researchers, and opens up the possibility of extending elastography, deconvolution, spectral processing and other RF analysis into 3D.
\end{abstract}

\section{Introduction}

The availability of radio frequency (RF) ultrasound (US) data enables the study of elastography 4], flow detection, deconvolution [2], spectral processing [3] and other important topics. Many of these require real-time acquisition, and all are considerably enhanced by the availability of 3D data. However, access to such data is currently limited: RF analysis is often based on single frames acquired at only 8-bits sampling resolution, with several minutes delay to download the data to an external PC [3]. A 3D RF system using 16-bit acquisition at $20 \mathrm{MHz}$ has been reported [2], but not in real-time. Similarly a real-time system using 12-bit acquisition at $30 \mathrm{MHz}$ has been reported 4, but not in 3D. Commercial $\mathrm{RF}$ systems are generally neither real-time nor 3D.

We present a real-time 3D RF system, using 14-bit (60 dB useful dynamic range) acquisition, synced to the US machine clock at $66.6 \mathrm{MHz}$, which is capable of recording 30-60 frames of $\mathrm{RF}$ data per second (fps). The softwart 1 [1 requires a Gage CompuScope 14100 PCI analogue to digital converter. Each frame is downloaded from on-board Gage memory to a $3 \mathrm{GHz}$ Pentium 4 PC in realtime, hence storage is only limited by PC RAM. The system will work with any ultrasound machine from which analogue focused RF data and some simple timing signals are available: we use a Dynamic Imaging Diasus US machine.

\section{Validation}

3D point precision of the non-RF system has been demonstrated as $0.6 \mathrm{~mm}$ [1]. A high sampling resolution is crucial to preserving signal-to-noise at depth, since the RF data is acquired before log-compression: Fig. 11(a) demonstrates a similar noise floor for B-scans derived from acquired RF data to those from the US machine. Repeatability of the RF phase from vector to vector is also critical for analysing 3D RF data. This was assessed at $2 \mathrm{~cm}$ depth by scanning a planar

\footnotetext{
* Graham Treece is supported by an EPSRC/RAEng Postdoctoral Fellowship

1 Stradx, freely available from http://mi.eng.cam.ac.uk/ ${ }^{\text {rwp }} /$ stradx/ 


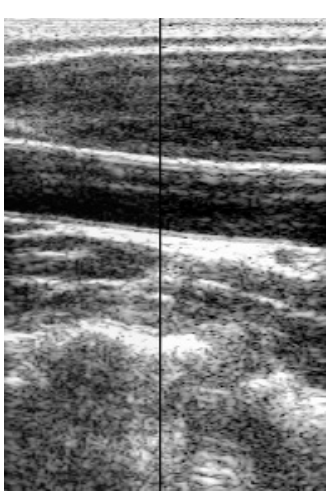

(a)

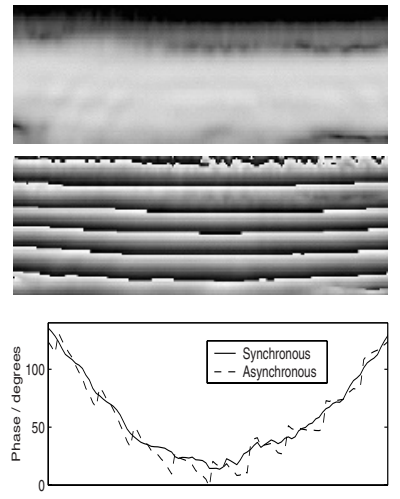

(b)

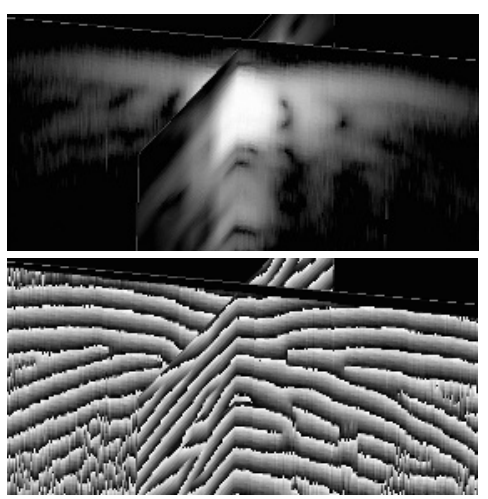

(c)

Fig. 1. (a) B-scan from US machine (left) and derived from RF data (right). (b) Bscan and phase image of a plane, magnified x20 axially, with corresponding intra-vector phase variation. (c) 3D RF amplitude and phase of the tip of a $0.15 \mathrm{~mm}$ wire, showing the elevational (left to right) and lateral (front to back) point spread function.

target with a 5-10 MHz probe, as in Fig. 11(b). The inter-vector phase had $2.3^{\circ}$ standard deviation at the centre frequency, compared to $8.0^{\circ}$ for asynchronous $100 \mathrm{MHz}$ sampled data. Intra-vector variation was similar, as shown in graphical form in Fig. 1(b). High accuracy 3D RF data is demonstrated in Fig. 11(c).

Speed of acquisition is an equally important factor. Data is transfered from the Gage card to the $\mathrm{PC}$ at $75 \mathrm{Mb} / \mathrm{s}$, hence for $66.6 \mathrm{MHz}$ sampling, every third frame can be stored. This typically gives full-frame rates of 30-60 fps, dependent on probe frequency, assuming a single transmit focus. This rate can be increased upwards of $1000 \mathrm{fps}$ if not all of the RF vectors are acquired. RF-derived B-scans can be displayed in real-time, though at a reduced frame rate of typically 15 fps.

\section{Conclusions}

We have presented a real-time 3D RF US system which is fast and accurate enough to form a solid basis for the study of RF US processing in 3D, yet available at only the cost of an acquisition card and a suitable US machine.

\section{References}

1. Treece, G.M., Gee, A.H., Prager, R.W., Cash, C.J.C., Berman, L.H.: High definition freehand 3D ultrasound. Ultrasound Med Biol 29 (2003) 529-546

2. Taxt, T.: Three-dimensional blind deconvolution of ultrasound images. IEEE T Ultrason Ferr 48 (2001) 867-871

3. Watson, R.J., McLean, C.C., Moore, M.P., Spencer, T., Salter, D.M., Anderson, T., Fox, K.A.A., McDicken, W.N.: Classification of arterial plaque by spectral analysis of in vitro RF intravascular ultrasound data. Ultrasound Med Biol 26 (2000) 73-80

4. Pesavento, A., Lorenz, A., Siebers, S., Emmert, H.: New real-time strain imaging concepts using diagnostic ultrasound. Phys Med Biol 45 (2000) 1423-1435 